\title{
EDITORIAL
}

\section{A step in the right direction}

\author{
Fighting infectious diseases in developing countries is difficult owing to limited financial \\ resources. Many pharmaceutical companies have programmes that provide much needed \\ medicines, but more can be done.
}

The recent announcement by GlaxoSmithKline (GSK) that it will make the structures of over 13,500 compounds from its antimalarial screening programme publicly available is another welcome example of a pharmaceutical company's support for research on diseases that affect developing nations. Under its 'Open Lab' structure, GSK will allow any interested party to develop these compounds further as antimalarials. Taking this one step further, GSK has pledged US\$8 million to provide up to 60 researchers with the opportunity to investigate these compounds at GSK's Tres Cantos campus in Madrid, Spain.

In recent years, the involvement of pharmaceutical companies in the development of treatments for infectious diseases affecting developing countries and in providing access to these medicines has increased. According to the International Federation of Pharmaceutical Manufacturers \& Associations, the pharmaceutical industry as a whole provided over $\$ 9.2$ billion in health interventions (such as medicines and equipment) between 2000 and 2007, reaching 1.75 billion people worldwide. However, there are large differences in the involvement of the various companies. A 2008 report from the Access to Medicine Foundation, a Netherlands-based organization that aims to advance access to health care in developing countries, provides an overview of the involvement of major pharmaceutical companies in such access programmes. The report reveals that some companies, such as GSK and Novo Nordisk, are leaders in providing access, whereas other companies, such as Eli Lilly, have taken a more targeted approach with their MDR-TB (multidrug-resistant tuberculosis) Partnership.

One of the ways that drug companies support developing nations is through drug donation programmes. Perhaps one of the best known is Merck's ivermectin (Mectizan) donation programme to fight river blindness; Merck has pledged to provide this drug free of charge until river blindness is eradicated. Since 1987, 2.5 billion tablets of Mectizan have been distributed, and as a result the spread of the disease has been halted in several regions worldwide. In addition, ivermectin is used by the Global Alliance to Eliminate Lymphatic Filariasis, in conjuction with albendazole, a drug that was donated by GSK. GSK has donated over one billion doses of albendazole and anticipates donating another five billion doses before the expected elimination of lymphatic filariasis in 2020. As a result of these efforts, an estimated 6.6 million children have been spared the disfigurement of this disease. Other donation programmes include the donation of medical equipment by Johnson \& Johnson to treat guinea worm disease, the donation of azithromycin (Zithromax) by Pfizer for the treatment of glaucoma and the Gardasil Access Programme from Merck, which will provide at least 3 million doses of the human papillomavirus vaccine to low-income countries.

However, donation programmes work best in limited circumstances, such as in disease eradication or elimination programmes or in response to natural disasters. Many companies promote access to medicines through alternative means, including pricing schemes that make medicines available at reduced (non-profit) prices. For example, Gilead provides the HIV drugs tenofovir disoproxil fumarate (Viread) and Truvada (which is a combination of tenofovir and emtricitabine) at prices that reflect the economic status of each country, and for the least developed countries GSK has instituted sustainable pricing for antiretrovirals, which allows them to cover the cost without making a profit. Another, equally important, way in which drugs and vaccines are made more affordable is the non-enforcement policy, whereby drug companies agree not to enforce patents in particular regions to allow companies in these regions to produce the drugs more cheaply, or they set up technology transfer agreements that allow companies in developing nations to produce generic versions. The technology transfer agreements not only provide lower-cost medications but also stimulate research and development in these regions.

Despite these efforts, the pharmaceutical companies fall far behind the Bill and Melinda Gates Foundation and the US National Institutes of Health in terms of research funding, and so it is crucial that companies remain committed to these programmes even during the economic downturn. Their current efforts are admirable and have reached many individuals who would not have had access to medicines otherwise. Much work remains to be done, however, and we hope that other companies follow GSK's lead in making available today the compounds that may be tomorrow's medicines. 